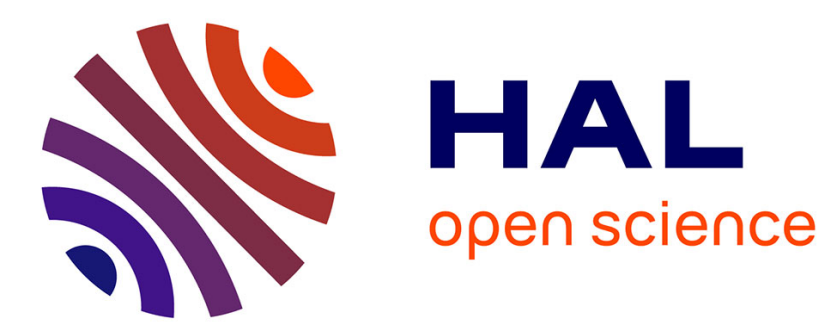

\title{
Mesure d'instabilités dans un plasma chaud et dense par diffusion d'ondes millimétriques
}

\author{
F. Koechlin, V. Glaude, J. How
}

\section{To cite this version:}

F. Koechlin, V. Glaude, J. How. Mesure d'instabilités dans un plasma chaud et dense par diffusion d'ondes millimétriques. Revue de Physique Appliquée, 1977, 12 (11), pp.1797-1803. 10.1051/rphysap:0197700120110179700 . jpa-00244404

\section{HAL Id: jpa-00244404 https://hal.science/jpa-00244404}

Submitted on 1 Jan 1977

HAL is a multi-disciplinary open access archive for the deposit and dissemination of scientific research documents, whether they are published or not. The documents may come from teaching and research institutions in France or abroad, or from public or private research centers.
L'archive ouverte pluridisciplinaire HAL, est destinée au dépôt et à la diffusion de documents scientifiques de niveau recherche, publiés ou non, émanant des établissements d'enseignement et de recherche français ou étrangers, des laboratoires publics ou privés. 


\title{
MESURE D'INSTABILITÉS DANS UN PLASMA CHAUD ET DENSE PAR DIFFUSION D'ONDES MILLIMÉTRIQUES (*)
}

\author{
F. KOECHLIN, V. GLAUDE (**) et J. HOW \\ Association EURATOM-CEA sur la Fusion, \\ Département de Physique du Plasma et de la Fusion Contrôlée \\ Centre d'Etudes Nucléaires, BP n ${ }^{0}$ 6, 92260 Fontenay-aux-Roses, France \\ (Reçu le 30 décembre 1976, accepté le 25 juillet 1977)
}

\begin{abstract}
Résumé. - Les possibilités de la diffusion d'ondes électromagnétiques pour mesurer les spectres de turbulence dans un plasma sont rappelées. On examine les conditions expérimentales à satisfaire pour réaliser une telle mesure dans un plasma donné. On présente ensuite la réalisation d'un dispositif de diffusion de micro-ondes de $2 \mathrm{~mm}(f \sim 136 \mathrm{GHz})$ qui a été utilisé pour mettre en évidence des instabilités de dérive $(f \sim 300 \mathrm{kHz})$ dans le plasma du Tokamak T. F. R.

Abstract. - The use of electromagnetic wave scattering techniques for measuring the spectra of plasma turbulence is discussed. The experimental constraints that must be satisfied for such a measurement in a given plasma are examined. Finally, an experiment is presented in which the scattering of $2 \mathrm{~mm}$ micro-waves $(f \sim 136 \mathrm{GHz})$ is used to investigate the universal drift instability $(f \sim 300 \mathrm{kHz})$ in the T. F. R. Tokamak.
\end{abstract}

1. Introduction. - La diffusion d'ondes électromagnétiques par les électrons d'un plasma [1] est un procédé maintenant largement répandu pour mesurer la densité et la température électroniques (diffusion Thomson), dès que le plasma est assez dense et chaud [2,3]. Cette diffusion est toujours produite par les fluctuations de la densité électronique. Dans le cas de la diffusion Thomson, on mesure les fluctuations de courte longueur d'onde - inférieure à la distance de Debye $\lambda_{\mathrm{D}}$ - qui proviennent de l'agitation thermique des électrons. Quand, au contraire, les fluctuations ont une échelle supérieure à $\lambda_{\mathrm{D}}$, elles sont associées à des phénomènes collectifs, du type onde. Dans ce dernier cas, les propriétés très particulières de la diffusion d'ondes e. m., analogue à la diffraction de Bragg dans les cristaux, permettent d'obtenir des informations très utiles sur les régimes d'ondes ou sur la turbulence dans un plasma $[4,5,6,7,8]$. Cette méthode d'investigation a déjà été largement employée dans des colonnes de plasma $[9,10,11,12]$ ou des expériences d'ondes de choc [13] pour étudier des phénomènes fondamentaux. Ses avantages essentiels sont de ne pas perturber le milieu (par l'introduction de sondes,

(*) Communication présentée au Congrès National des Plasmas, 6-10 décembre 1976.

(**) Adresse actuelle : Université de Montréal. Canada. par exemple), et de recueillir une information concernant le cour même du plasma. D'ailleurs, lorsqu'on étudie un plasma à grande densité d'énergie thermique, comme c'est le cas aujourd'hui dans la recherche pour la fusion thermonucléaire, seules des méthodes d'investigation par faisceaux d'ondes ou de particules restent possibles.

Cette communication rappelle d'abord les propriétés de la diffusion d'onde électromagnétiques par des fluctuations de densité électronique ; puis les conditions expérimentales nécessaires pour qu'une mesure de modes collectifs de fluctuations puisse être réalisée. On examine, à titre d'application, les problèmes posés par la mesure de la turbulence associée à des instabilités de dérive dans le plasma d'un Tokamak $[14,15]$. Les caractéristiques de ces instabilités, du plasma et de la machine Tokamak ont nécessité le développement d'un appareillage original : un récepteur hétérodyne à $\lambda=2 \mathrm{~mm}(136 \mathrm{GHz})$ asservi en fréquence avec l'émetteur éclairant le plasma. Ce dispositif est décrit dans la dernière partie de l'article.

2. La méthode de mesure. - Lorsqu'un faisceau d'ondes e. $\mathrm{m}$. de vecteur d'onde $k_{0}\left(=\omega_{0} / c\right)$ se propage dans un plasma d'indice $v$ voisin de 1 $\left(\omega_{0} \gg \omega_{\mathrm{p}}\right.$, la fréquence plasma), une petite fraction du flux de photons est diffusée par les fluctuations de densité électronique $\tilde{n}(r, t)\left(=n_{\mathrm{e}}(r, t)-\bar{n}: \bar{n}\right.$ est 
la densité moyenne, à variations lentes). Par suite des lois de conservation de l'énergie et de la quantité de mouvement, une composante de Fourier $(\Omega, \mathbf{K})$ des fluctuations de densité donnera naissance à deux ondes diffusées de fréquences et de directions différentes (Fig. 1) :

$\left(\omega_{0}, \mathbf{k}_{0}\right)$ onde incidente

$(\Omega, \mathbf{K})$ onde de densité

$$
\begin{aligned}
& \left(\omega_{1}=\omega_{0}+\Omega, \mathbf{k}_{1}=\mathbf{k}_{0}+\mathbf{K}\right) \\
& \left(\omega_{2}=\omega_{0}-\Omega, \mathbf{k}_{2}=\mathbf{k}_{0}-\mathbf{K}\right)
\end{aligned}
$$

ondes diffusées.

Les ondes diffusées contiennent donc l'information $(\Omega, \mathbf{K})$ et peuvent la transmettre à l'extérieur du plasma $[16,17]$.

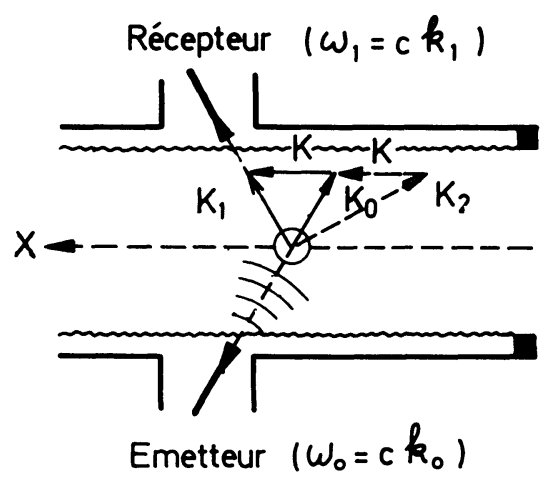

FIG. 1. - Diffusion d'une onde électromagnétique $\left(\omega_{0}, k_{0}\right)$ par des fluctuations de densité $(\Omega, K)$ se propageant dans la direction $x$.

[Scattering of an electromagnetic wave $\left(\omega_{0}, k_{0}\right)$ by density fluctuations $(\Omega, K)$ propagating in the direction $x$.]

Dans la suite, nous ne considérerons, pour simplifier, que l'une des deux ondes diffusées.

Si $K \lambda_{\mathrm{D}}>1$, les fluctuations, dues à l'agitation thermique des électrons, donnent lieu à la diffusion Thomson. Nous ne discuterons pas ce cas ici.

Si au contraire $K \lambda_{\mathrm{D}}<1$, les composantes $(\Omega, \mathbf{K})$ des fluctuations de densité électronique sont associées à des modes collectifs - ondes électrostatiques ou magnétosoniques - du plasma. L'amplitude de ces fluctuations peut être fixée par l'équilibre thermodynamique, dans un plasma calme; c'est notamment le cas des ondes sonores engendrées par le mouvement thermique des ions. Leur niveau peut être beaucoup plus élevé lorsque ces fluctuations sont associées à des instabilités, résultant d'un écart à l'équilibre thermodynamique: distributions de vitesses non maxwelliennes ou anisotropes, inhomogénéités de densité ou de température, etc...

La valeur de la puissance disponible dans une des ondes e. $\mathrm{m}$. diffusées est reliée au flux d'énergie du rayonnement incident $\varepsilon_{0} c\left|E_{0}\right|^{2}$ par la relation suivante :

$$
\begin{aligned}
\mathrm{d} \boldsymbol{P}_{1}=\varepsilon_{0} c \mid \mathbf{u} \times(\mathbf{u} & \left.\times \mathbf{E}_{0}\right)\left.\right|^{2} r_{0}^{2}\left(\omega_{1} / \omega_{0}\right)^{2} \times \\
& \times \bar{n} V(2 \pi)^{3} S(\mathbf{K}, \Omega) \mathrm{d} \omega_{1} \mathrm{dO}
\end{aligned}
$$

dans laquelle $(\Omega, \mathbf{K}),\left(\omega_{1}, \mathbf{k}_{1}\right)$ et $\left(\omega_{0}, \mathbf{k}_{0}\right)$ satisfont aux relations (1), $\varepsilon_{0}$ est la permittivité du vide, $\mathbf{u}$ un vecteur unité dans la direction d'observation $\mathbf{k}_{1}$, $r_{0}$ le rayon de l'électron $\left(r_{0}=2,8 \times 10^{-13} \mathrm{~cm}\right), \bar{n}$ est la densité moyenne dans le volume $V$ où la diffusion prend place, dO est l'angle solide d'observation du rayonnement diffusé, $\mathrm{d} \omega_{1} / 2 \pi$ est la bande passante du récepteur employé. Enfin $\bar{n} S(\mathbf{K}, \Omega)$ est la densité spectrale des fluctuations de densité électronique de fréquence $\Omega=\omega_{1}-\omega_{0}$, et de vecteur d'onde

$$
\mathbf{K}=\mathbf{k}_{\mathbf{1}}-\mathbf{k}_{\mathbf{0}} .
$$

La valeur quadratique moyenne des fluctuations de densité lui est reliée par la relation :

$$
<|\tilde{n}(r, t)|^{2}>=\bar{n} \int S(\mathbf{K}, \Omega) \mathrm{d}^{3} \mathbf{K} \mathrm{d} \Omega
$$

issue du théorème de Wiener-Khintchine.

La puissance diffusée est donc proportionnelle au flux de puissance incident et au nombre $\bar{n} V$ d'électrons diffusants multiplié par leur section efficace $r_{0}^{2} \sim 10^{-25} \mathrm{~cm}^{2}$. Cette puissance est très faible dans le cas des fluctuations thermiques pour lesquelles

$$
(2 \pi)^{3} \int S(\mathbf{K}, \Omega) \mathrm{d} \Omega=1 ;
$$

ce dernier facteur peut au contraire devenir très grand dans le cas d'une turbulence, d'après (3). Dans un plasma magnétisé, où $\mathbf{B}$ est l'induction magnétique, l'expression (2) reste valable si $\mathbf{E}_{0} \times \mathbf{B}=0$.

Une bonne expérience de diffusion d'ondes e. $\mathrm{m}$. peut donc fournir, par la mesure de la puissance diffusée en fonction de la fréquence et de la direction, les spectres de fréquence et de nombre d'ondes des fluctuations. De plus, si la fonction de 4 variables $S(\mathbf{K}, \Omega)$ peut être complètement mesurée, la valeur absolue des fluctuations de densité est obtenue par (3). La connaissance de la relation de dispersion, ou même des hypothèses assez simples sur la nature des fluctuations observées permettent alors de calculer le potentiel électrostatique fluctuant $\tilde{\varphi}(r, t)$ et d'autres grandeurs associées. Cependant, cette méthode de mesure ne fournit pas la phase entre les diverses grandeurs associées à une onde, car on ne mesure que la valeur quadratique moyenne des fluctuations de densité.

3. Conditions pratiques d'une mesure. - A quelles conditions une mesure de fluctuations résultant d'une instabilité électrostatique peut-elle être réalisée dans un plasma de laboratoire? Nous prenons comme exemple les caractéristiques du plasma du dispositif T. F. R. [18] (Tokamak de Fontenay-aux-Roses) dans lequel des mesures ont été effectivement faites par les auteurs [15].

Les faisceaux d'ondes e. $m$. utilisés et la configuration de l'expérience doivent satisfaire aux conditions suivantes : 
$1^{\circ}$ Le plasma doit être transparent pour le rayonnement employé, et son indice de réfraction doit rester voisin de 1 pour avoir une propagation quasi rectiligne. Il faut donc :

$$
\omega_{0}, \omega_{1} \gg \omega_{\mathrm{pmax}}
$$

où $\omega_{\text {pmax }}^{2}=e^{2} \bar{n}_{\max } / \varepsilon_{0} m$. Dans le T. F. R., la densité centrale $\bar{n}_{\max }$ est dans la gamme $0,4 \div 1 \times 10^{14} \mathrm{~cm}^{-3}$, donc $\omega_{\text {pmax }} / 2 \pi \simeq 60 \div 90 \mathrm{GHz}$, et $\omega_{0,1} / 2 \pi$ doivent largement dépasser $90 \mathrm{GHz}$. Pour avoir toujours un indice $v \geqslant 0,95$ au centre, il faudrait

$$
\omega_{0} / 2 \pi \geqslant 280 \mathrm{GHz} \text {. }
$$

$2^{\circ}$ Pour mesurer les modes collectifs du plasma, qui satisfont à $K \lambda_{\mathrm{D}}<1$, on doit avoir en pratique :

$$
K \lambda_{\mathrm{D}}=\left|\mathbf{k}_{1}-\mathbf{k}_{0}\right| \lambda_{\mathbf{D}} \leqslant 0,5
$$

où $\lambda_{\mathrm{D}}^{2}=\varepsilon_{0} T_{\mathrm{e}} / \bar{n} e^{2}$. Dans le plasma du Tokamak T. F. R., dont les profils typiques de densité et de température électroniques sont donnés sur la figure 2 ,

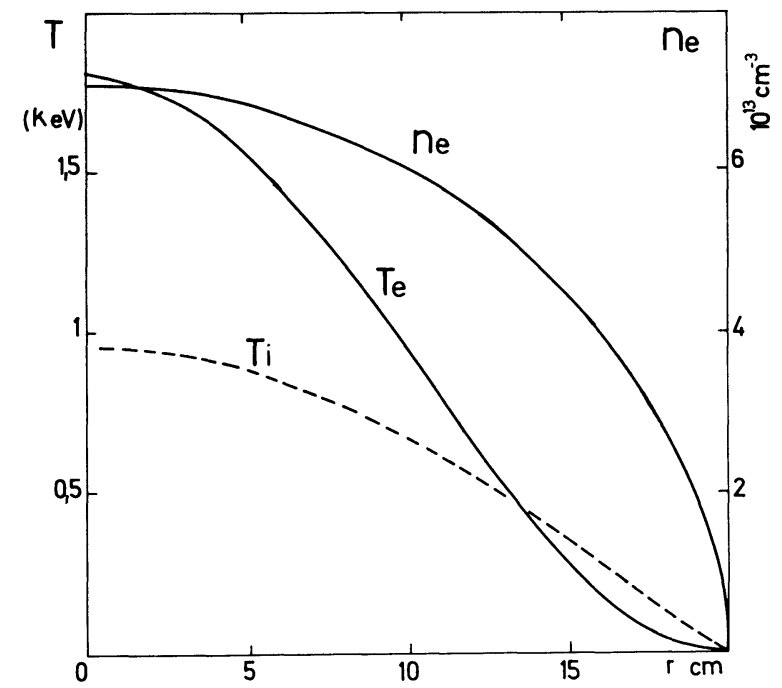

Fig. 2. - Profils typiques de densité et de température électroniques $\left(n_{\mathrm{e}}, T_{\mathrm{e}}\right)$ et ionique $\left(T_{\mathrm{i}}\right)$ dans un Tokamak.

[Typical profiles of electron density and temperature $\left(n_{\mathrm{e}}, T_{\mathrm{e}}\right)$ and of ion temperature $\left(T_{\mathrm{i}}\right)$ in a Tokamak.]

$\lambda_{\mathbf{D}}$ reste comprise entre 20 et $50 \mu \mathrm{m}$, et la borne supérieure de $K=\left|\mathbf{k}_{1}-\mathbf{k}_{0}\right|$ est d'environ $100 \mathrm{~cm}^{-1}$. Dans le cas, le plus fréquent, où la fréquence des fluctuations satisfait à $\Omega \ll \omega_{\mathrm{p}}$, utilisant (4) et (1) on voit que $\left(\omega_{1}-\omega_{0}\right) / \omega_{0} \ll 1$, donc

$$
\left|\mathbf{k}_{1}\right| \sim\left|\mathbf{k}_{0}\right|\left(\sim \omega_{0} / c\right) .
$$

Il en résulte que :

$$
\left|\mathbf{k}_{1}-\mathbf{k}_{0}\right| \sim 2 k_{0} \sin \theta / 2
$$

ne dépend que de l'angle $\theta$ fait par le rayonnement diffusé avec la direction du rayonnement incident. La condition (5) devient alors :

$$
2 \sin \theta / 2 \leqslant 0,5 / k_{0} \lambda_{\mathrm{D}}\left(=\lambda_{0} / 4 \pi \lambda_{\mathrm{D}}\right) .
$$

TABLEAU I

Source

Laser $\mathrm{CO}_{2}$

Laser $\mathrm{CH}_{3} \mathrm{OH}$

Laser $\mathrm{HCN}$

Carcinotron de rayonnement

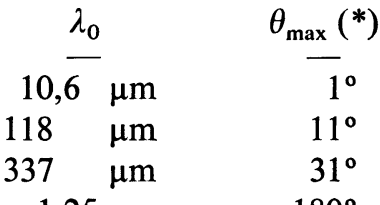

$$
\begin{aligned}
& 1,25 \mathrm{~mm} \quad 180^{\circ}
\end{aligned}
$$

(*) Relation (7) avec $\lambda_{\mathrm{D}}=50 \mu \mathrm{m}$.

L'angle $\theta$ maximum pour des ondes diffusées par des modes collectifs est donné dans le tableau I, pour quelques longueurs d'ondes $\lambda_{0}$ typiques des générateurs possibles. La dernière ligne du tableau donne la longueur d'onde

$$
\lambda_{0}^{*}=8 \pi \lambda_{\mathrm{D}}
$$

pour laquelle $\theta_{\max }=180^{\circ}\left(\omega_{0}^{*} / 2 \pi=240 \mathrm{GHz}\right)$. Pour une longueur d'onde $\lambda_{0}>\lambda_{0}^{*}$, la condition (7) sera satisfaite pour tous les angles, mais la gamme des modes collectifs mesurables sera limitée à

$$
0 \leqslant K \leqslant 2 k_{0}\left(<0,5 / \lambda_{\mathrm{D}}\right) .
$$

$3^{\circ}$ Supposons que l'on désire mesurer des fluctuations dont les vecteurs d'ondes $\mathbf{K}\left(=\mathbf{k}_{1}-\mathbf{k}_{0}\right)$ ont pu être estimés, par exemple par des considérations théoriques, et dont la fréquence $\Omega$ est très inférieure à $\omega_{\mathrm{p}}$. La relation (6) montre alors que l'angle de diffusion est une fonction croissante de la longueur d'onde porteuse $\lambda_{0}=2 \pi / k_{0}$. Cette longueur d'onde pourra donc être choisie pour donner à l'angle $\theta$ une valeur raisonnable dans l'expérience projetée. Or l'emploi de grands angles de diffusion est souhaitable pour deux raisons. En premier lieu, la puissance du rayonnement diffusé étant très faible, il est important d'éliminer au maximum l'entrée du rayonnement incident dans un récepteur sensible. D'autre part, la résolution spatiale de la mesure, déterminée par l'intersection d'un faisceau incident et d'un cône d'observation (Fig. 4), est d'autant meilleure que l'angle $\theta$ est plus grand.

$4^{\circ}$ Des raisons intrinsèques à la méthode limitent la résolution spatiale de la mesure. En effet, les relations (1) ne sont vérifiées qu'à une certaine approximation $\Delta K \sim \pi V^{-1 / 3}$, où $V$ est le volume défini par l'intersection des deux faisceaux. Une bonne résolution dans la mesure de $K$ implique donc que les dimensions du volume de diffusion soient beaucoup plus grandes que la longueur d'onde des fluctuations.

La synthèse des conditions à remplir pour mesurer des modes collectifs par diffusion montre que, pour un plasma donné, le meilleur choix de l'onde e. $\mathrm{m}$. porteuse $\left(\omega_{0}, \mathbf{k}_{0}\right)$ est un compromis entre la fréquence la plus élevée possible $\left(1^{\circ}\right)$ et une longueur d'onde suffisamment grande $\left(2^{\circ}, 3^{\circ}\right)$. Dans le cas du plasma d'un Tokamak $\left(\bar{n} \sim 0,5 \times 10^{14} \mathrm{~cm}^{-3} ; T_{\mathrm{e}} \leqslant 2 \mathrm{keV}\right)$, 
le choix d'une fréquence de 150 à $300 \mathrm{GHz}\left(\lambda_{0} \lesssim 2 \mathrm{~mm}\right)$ satisfait bien aux conditions (4) et (7). Ce résultat met donc en relief l'intérêt des micro-ondes pour les mesures de fluctuations dans les plasmas confinés qui existent actuellement.

4. Mesure d'ondes de dérive dans le Tokamak T. F. R. - L'objectif qui était proposé aux auteurs était de mettre en évidence l'existence d'ondes de dérive dans le plasma du T. F. R., de mesurer leurs spectres - fréquence et vecteur d'ondes - afin de les comparer aux modèles théoriques, et d'évaluer leur amplitude, définie selon (3), en vue d'en déduire des coefficients de transport turbulent. Nous n'exposerons ici que les données du problème posé au diagnostic de diffusion.

Les ondes de dérive, instables en présence d'un gradient de densité (et dans certains cas, de température) doivent avoir les propriétés suivantes :

- propagation quasi normale au champ magnétique avec une vitesse de phase voisine de la vitesse diamagnétique des électrons $V_{\text {de }}$ (Fig. 3);

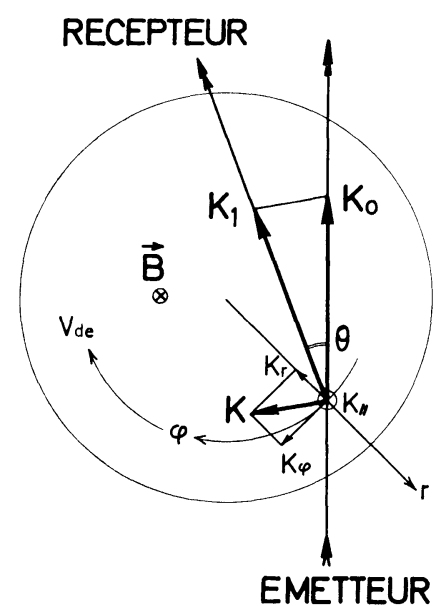

Fig. 3. - Section droite du Tokamak. Schéma physique d'une expérience de diffusion sur des modes de dérive. $\left(B_{\mathrm{T}}:\right.$ champ magnétique toroïdal. $V_{\mathrm{de}}$ : vitesse diamagnétique des électrons.) [Minor cross-section of a Tokamak. Schematic of a scattering experiment to detect drift waves. $\left(B_{\mathrm{T}}\right.$ : Toroidal magnetic field. $V_{\text {de }}:$ diamagnetic electron velocity.)]

- nombre d'ondes perpendiculaires au champ $K_{\mathrm{T}}$ vérifiant $K_{\mathrm{T}} a_{\mathrm{i}} \sim 1 . a_{\mathrm{i}}$ est le rayon de Larmor des ions, $a_{\mathrm{i}}=\left(T_{\mathrm{i}} M\right)^{1 / 2} / e B$ (Fig. 2) avec $B=4$ à $5 \mathrm{~T}$.

Compte tenu des caractéristiques du plasma décrit en figure 2, les paramètres à mesurer sont les suivants :

$$
\begin{aligned}
\Omega / 2 \pi & =30 \div 200 \mathrm{kHz}, \quad K_{\varphi}=6 \div 15 \mathrm{~cm}^{-1}, \\
K_{\mathrm{r}} & =2 \div 6 \mathrm{~cm}^{-1}, \quad K_{\prime \prime} \sim 10^{-2} \mathrm{~cm}^{-1} .
\end{aligned}
$$

Afin d'augmenter les angles de diffusion, et compte tenu des possibilités de la technique, nous avons choisi l'emploi d'ondes de $2,2 \mathrm{~mm}$

$$
\left(\omega_{0} / 2 \pi=136 \mathrm{GHz} ; \quad k_{0}=28 \mathrm{~cm}^{-1}\right) .
$$

Nous exposons maintenant les problèmes qu'il a fallu résoudre et les solutions adoptées sur l'appareillage qui a servi à faire les mesures [15].

4.1 Propagation des ondes électromagnétiques. - Si le plasma est dit transparent pour un rayonnement dont la fréquence satisfait à (4), il n'est pas forcément exempt de réfraction. En effet, avec une fréquence de $136 \mathrm{GHz}$ et une densité

$$
\bar{n}_{\max }=0,7 \times 10^{14} \mathrm{~cm}^{-3} \quad \text { (Fig. 2), }
$$

l'indice

$$
v=\left(1-\omega_{\mathrm{p}}^{2} / \omega_{0}^{2}\right)^{1 / 2}
$$

vaut 0,7 au centre du plasma. Utilisant des travaux antérieurs [20,21], nous avons développé un code numérique qui calcule la propagation de faisceaux d'ondes électromagnétiques dans un milieu inhomogène à symétrie cylindrique. Dans le cas où le profil radial de densité est parabolique, le code calcule le point d'intersection de deux faisceaux, leur angle, les composantes de $\mathbf{k}_{1}-\mathbf{k}_{0}$, le volume d'interaction. Ce code a permis d'interpréter les mesures faites sur le T. F. R.

4.2 RéSOlution ANGUlaire ET SPatiale. - D'après la relation (6) le rayonnement diffusé fait avec le rayonnement incident les angles suivants :

$-\theta \sim 10^{-2}$ degré dans la direction parallèle au champ magnétique ;

$-12^{\circ} \leqslant \theta \leqslant 30^{\circ}$ pour $K_{\varphi}$ et $4^{\circ} \leqslant \theta \leqslant 10^{\circ}$ pour $K_{\mathrm{r}}$ (Fig. 3).

Il est donc exclu de mesurer $K_{\| \prime}$ avec la résolution angulaire d'un faisceau de micro-ondes. En revanche, $K_{\mathrm{r}}$ et $K_{\varphi}$ sont mesurables, à condition d'avoir une résolution angulaire meilleure que $4^{\circ}$. Nous avons, dans ce but, réalisé des cornets rectangulaires très directifs dont les caractéristiques sont données dans le tableau II. Nous avons effectivement pu mesurer des vecteurs d'ondes de $4 \mathrm{~cm}^{-1}$, sans être gênés par la lumière parasite.

\section{TABLEAU II}

$$
\text { Cornet de haute directivité }\left({ }^{1}\right) \text { à } \lambda_{0}=2,2 \mathrm{~mm}
$$

$\begin{array}{ccccc} & \begin{array}{c}\text { Largeur de } \\ \text { l'embou- } \\ \text { chure }\end{array} & \begin{array}{c}1 / 2 \text { angle } \\ \text { des parois }\end{array} & 1 / 2 \text { angle à } & 1 / 2 \text { angle à } \\ & -\overline{d B}(2) & -10 \mathrm{~dB}(2) \\ \text { Plan E } & 20 \mathrm{~mm} & 3^{\circ} 10^{\prime} & 1^{\circ} 45^{\prime} & 3^{\circ} 10^{\prime} \\ \text { Plan H } & 28 \mathrm{~mm} & 4^{\circ} & 1^{\circ} 45^{\prime} & 3^{\circ} 10^{\prime}\end{array}$

(1) Gain : 28,5 B

(2) Mesurés à $90 \mathrm{~cm}$ du sommet du cornet.

La disposition géométrique des faisceaux est montrée sur la figure 4 ; elle est largement tributaire des accès standards du T. F. R. et ne permet pas de 

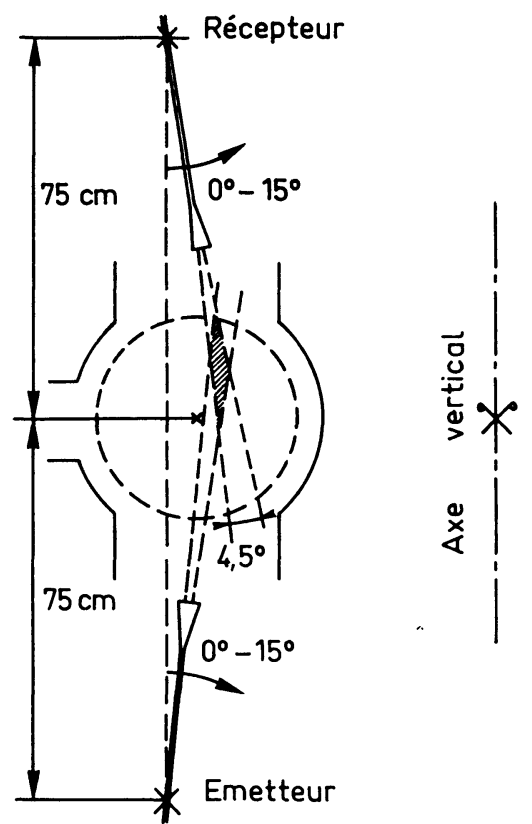

Fig. 4. - Disposition des faisceaux d'ondes millimétriques employés pour la mesure de modes de dérive sur le Tokamak T. F. R.

[Arrangement of the millimetre wave beams used to measure drift waves in the T. F. R. Tokamak.]

mesurer les spectres de $K$ en un point fixe du plasma en faisant pivoter les faisceaux autour de ce point [12, 19]. Cependant, en variant les inclinaisons de l'émetteur et du récepteur, l'angle fait par les faisceaux - donc l'amplitude et la direction de $\mathbf{K}=\mathbf{k}_{1}-\mathbf{k}_{0}$ varie, pendant que le volume d'interaction décrit la section droite du plasma. Disposant de deux paramètres, il est donc possible de balayer le plasma en remplissant une condition : $|K|=$ Cte ou $K_{\mathrm{r}}=$ Cte, ou $r=$ Cte par exemple. On peut ainsi obtenir séparément les spectres de nombres d'ondes $S\left(K_{\mathrm{r}}\right)$ et $S\left(K_{\varphi}\right)$ à un rayon constant, et la variation radiale des fluctuations à nombre d'ondes constant.

4.3 RÉSOLUTION EN FRÉQUENCE. - La densité élevée du plasma du T. F. R. impose l'emploi d'un rayonnement micro-ondes de fréquence

$$
F_{0}\left(=\omega_{0} / 2 \pi\right) \sim 140 \mathrm{GHz},
$$

alors que les fréquences prévues pour les modes de dérive - typiquement $100 \mathrm{kHz}$ - sont faibles. Il en résulte que le décalage en fréquence du rayonnement diffusé est très petit devant la fréquence de la source : $\Omega / \omega_{0} \sim 10^{-6}$. D'autre part, le niveau très faible du rayonnement diffusé nécessite l'emploi d'un récepteur à faible bruit, c'est-à-dire d'un récepteur hétérodyne ayant une moyenne fréquence d'au moins quelques dizaines de $\mathrm{MHz}$; ce type de récepteur a d'ailleurs un autre avantage, décrit plus loin.

La figure 5 représente schématiquement les fréquences des ondes mises en jeu dans l'expérience, et la figure 6 décrit l'émetteur, le récepteur et leurs connexions. D'après (1), le rayonnement reçu à la

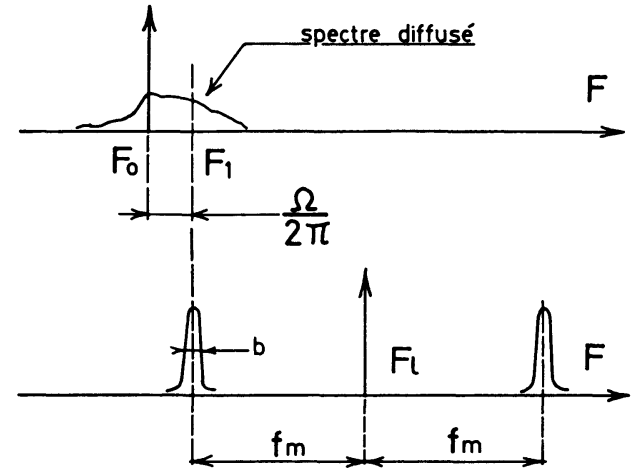

Fig. 5. - Positions relatives des fréquences employées dans la mesure : Axe supérieur : rayonnement reçu du plasma $\left(F_{0}\right.$ : fréquence de la source, $F_{1}$ : onde diffusée). Axe inférieur : fréquences présentes au niveau du récepteur $\left(F_{\mathrm{L}}\right.$ : oscillateur local, $f_{\mathrm{m}}$ : moyenne fréquence).

[Relative positions of the frequencies used in the experiment. Top line : Scattered Radiation received from the plasma $\left(F_{0}\right.$ : incident wave, $F_{1}:$ scattered wave). Bottom line : frequencies seen at the level of the receiver $\left(F_{\mathrm{L}}\right.$ : local oscillator, $f_{\mathrm{m}}:$ intermediate frequency).]

fréquence $F_{1}$ provient des fluctuations de densité à la fréquence $\Omega / 2 \pi=F_{1}-F_{0}$; dans le récepteur le battement avec l'oscillateur local $F_{\mathbf{L}}$ produit une moyenne fréquence $f_{\mathrm{m}}=F_{\mathrm{L}}-F_{1}=F_{\mathrm{L}}-F_{0}-\Omega / 2 \pi$, voisine de $60 \mathrm{MHz}$. Si l'on désire mesurer $\Omega$ avec une précision de $10 \%, F_{\mathrm{L}}, F_{0}$ et $f_{\mathrm{m}}$ doivent être connues à mieux que $10 \mathrm{kHz}$. C'est le cas de $f_{\mathrm{m}}$, définie à $\sim 1 \mathrm{kHz}$ par un analyseur de spectres ou un filtre ; au contraire, les fréquences $F_{\mathrm{L}}$ et $F_{0}$ des klystrons millimétriques utilisés $\left({ }^{1}\right)$ ont des fluctuations permanentes de l'ordre de la dizaine de $\mathrm{kHz}$. Cependant, au moyen d'une boucle d'asservissement, représentée sur la figure 6, il est possible de maintenir constante

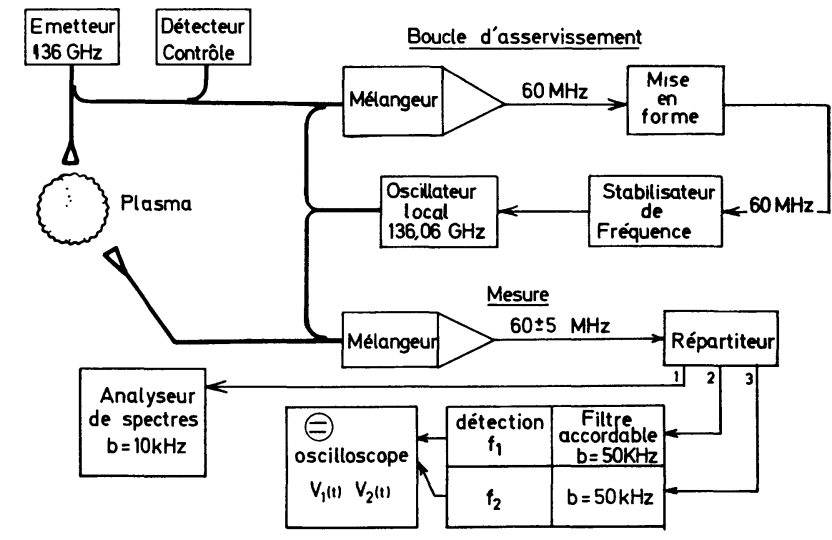

FIG. 6. - Schéma du dispositif émetteur-récepteur hétérodyne avec asservissement de fréquence à $136 \mathrm{GHz}$.

[Schematic of the complete heterodyne system at $136 \mathrm{GHz}$ with phase locking.]

(1) Un carcinotron de puissance $1 \mathrm{~W}$ a aussi été utilisé comme source dans les mêmes conditions. 
à $\sim 100 \mathrm{~Hz}$ près la différence $F_{\mathrm{L}}-F_{0}(=60 \mathrm{MHz})$; ceci suffit pour mesurer $\Omega$ avec la précision recherchée.

L'analyse du spectre de turbulence du plasma est alors réalisée en variant la moyenne fréquence $f_{\mathrm{m}}$ au niveau de l'analyseur de spectre placé en fin de chaîne (voie 1). En effet, le préamplificateur a une large bande passante $(10 \mathrm{MHz})$, et la bande passante de la mesure est fixée par le filtre de l'analyseur $(1 \mathrm{kHz}$ à $10 \mathrm{kHz}$ ), qui permet ainsi d'atteindre une précision de $1 \mathrm{kHz}$ dans la mesure du spectre de fréquence. Celui-ci est obtenu pendant un balayage d'une durée de $30 \mathrm{~ms}$, à un instant convenablement choisi au cours d'une décharge du Tokamak, dont la durée est de 250 à $500 \mathrm{~ms}$. Les voies 2 et 3 sont utilisées pour mesurer pendant toute la durée de la décharge les variations de la puissance diffusée à deux fréquences judicieusement choisies dans le spectre des fluctuations ; le nombre de voies de ce type n'est d'ailleurs pas limité.

Enfin, la sensibilité du récepteur à $136 \mathrm{GHz}$ a été évaluée au moyen d'une source monochromatique de puissance connue: on peut mesurer un signal de $2 \times 10^{-12} \mathrm{~W}$ avec une bande passante de $10 \mathrm{kHz}$.

Cette sensibilité, avec une source de rayonnement d'une puissance de $200 \mathrm{~mW}$, est suffisante pour mesurer une turbulence qui modifierait appréciablement les coefficients de transport du plasma $\left(\tilde{n} / \bar{n} \geqslant 10^{-4}\right)$.

4.4 RÉSULTAT EXPÉRIMENTAL. - La figure 7 montre deux spectres. Le premier est obtenu dans l'installation T. F. R. en l'absence du plasma, les faisceaux faisant un angle d'environ $15^{\circ}$; c'est la lumière parasite provenant de la source, qui est ramenée à $60 \mathrm{MHz}$ dans le récepteur. Le second est un spectre typique des fluctuations de densité de basse fréquence qui sont observées dans le plasma du T. F. R. (conditions de la figure 2), pour $K_{\mathrm{r}}<K_{\varphi} \sim 10 \mathrm{~cm}^{-1}$, et plutôt vers le bord du plasma $\left(r / r_{\max } \geqslant 0,7\right)$.

On remarque l'aspect dissymétrique du spectre : aux fréquences supérieures à celle de la source $(\Omega>0)$ correspondent des fluctuations plus intenses qu'aux fréquences inférieures à celles de la source $(\Omega<0)$. Cela signifie que les fluctuations observées se propagent préférentiellement dans la direction du vecteur d'onde $\mathbf{K}_{\varphi}$, qui est déterminée par la disposition géométrique de l'appareillage. Il se trouve que c'est le sens de la vitesse diamagnétique des électrons, élément d'interprétation important en faveur des ondes de dérive. Un tel résultat n'aurait pas pu être obtenu en employant un dispositif homodyne [14, 19] (dans lequel la source aurait aussi servi d'oscillateur local) $\left.\mathbf{(}^{2}\right)$.

L'ensemble des résultats physiques, concernant les

(2) Un dispositif homodyne a un autre inconvénient : il est sensible, comme un interféromètre, à la phase de l'onde reçue, c'est-à-dire à l'intégrale des variations de densité du plasma sur les trajets des faisceaux.

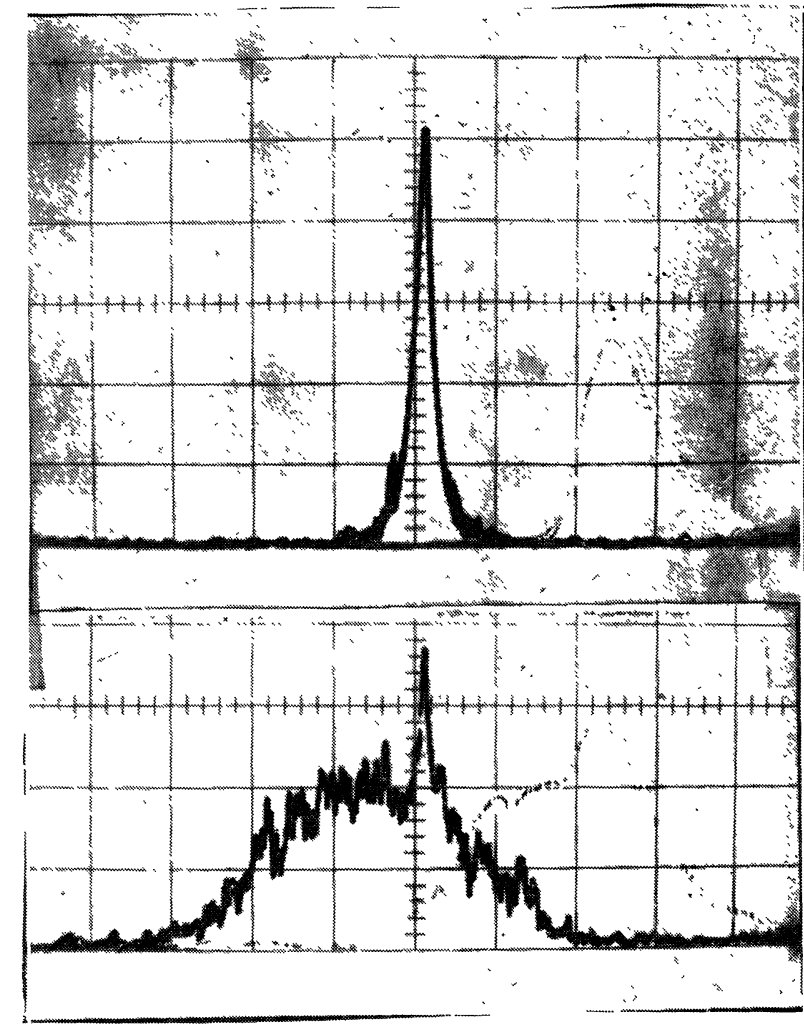

FIG. 7. - Analyse spectrale du rayonnement reçu sur le Tokamak (Horizontal : $300 \mathrm{kHz} / \mathrm{cm}$; Vertical : $8 \mathrm{~dB} / \mathrm{cm}$; la fréquence croît de droite à gauche): Haut : rayonnement parasite provenant de la source (sans plasma). Bas : spectre des fluctuations de densité dans les plasmas de la figure 2 . $\left(K_{\mathrm{r}} \sim 3 \mathrm{~cm}^{-1}, K_{\varphi} \sim 10 \mathrm{~cm}^{-1}, r \sim 16 \mathrm{~cm}\right.$.)

[Spectrum analysis of scattered radiation received from the Tokamak (Horizontal : $300 \mathrm{kHz} / \mathrm{cm}$; Vertical : $8 \mathrm{~dB} / \mathrm{cm}$; the frequency grows from right to left). Top : Parasitic radiation from the microwave source (without the plasma). Bottom : Density fluctuation spectrum for the plasma shown in figure 2 $\left(K_{\mathrm{r}} \simeq 3 \mathrm{~cm}^{-1}, K_{\varphi} \simeq 10 \mathrm{~cm}^{-1}, r \simeq 16 \mathrm{~cm}\right)$.]

fluctuations observées dans le T. F. R. et leurs effets sur les coefficients de transport des électrons, a été publié de façon préliminaire dans la référence [15]. Une publication détaillée est en cours de préparation.

5. Conclusion. - Un dispositif de diffusion d'ondes millimétriques a été construit et utilisé avec succès pour mesurer les spectres de turbulence de basse fréquence sur un Tokamak. De nombreuses difficultés ont été surmontées : difficultés d'accès au plasma, fréquence de la turbulence $10^{6}$ fois plus faible que celle des ondes millimétriques, source de faible puissance, enfin puissance diffusée $10^{7}$ fois plus faible, sous de petits angles par rapport au faisceau incident.

La discussion et les résultats montrent que la diffusion d'ondes électromagnétiques est un diagnostic riche en informations et susceptible d'être employé dans un grand nombre d'expériences sur les plasmas.

Remerciements. - Les auteurs tiennent à remercier MM. Aymar et Samain pour leur constant encouragement au cours de ce travail, MM. Gratadour et 
Patris pour leur concours efficace à la réalisation complète de l'expérience, et tous les membres de l'équipe T. F. R. pour leur aide amicale pendant la phase d'exploitation et d'interprétation.

\section{Bibliographie}

[1] Voir par exemple : Landau, L. D. et Lifshitz, E. M., Théorie des Champs (Edition de la Paix, Moscou) 1965.

[2] Fiocco, G. et Thompson, E., Phys. Rev. Lett. 10 (1963) 89.

[3] Charet, M., Dumay, L., de Michelis, C., Platz, P., Rapport EURATOM-CEA-FC-759 (1975) (Association-EURATOM-CEA, Fontenay-aux-Roses, France).

[4] Villars, F. et Weisskopf, V. F., Proc. IRE 43 (1955) 1232.

[5] Dougherty, J. P. et Farley, D. T., Proc. Roy. Soc. A 259 (1960) 79.

[6] Salpeter, E. E., Phys. Rev. 120 (1960) 1528.

[7] Fejer, J. A., Can J. Phys. 38 (1960) 1114.

[8] Rosenbluth, M. N. et Rostoker, N., Phys. Fluids 5 (1962) 776.

[9] Stern, R. A. et Tzoar, N., Phys. Rev. Lett. 15 (1965) 485.

[10] Arunassalam, V. and Brown, S. C., Phys. Rev. 140 (1965) A 471.

[11] BöHmer, H. and Raether, M., Phys. Rev. Lett. 16 (1966) 1145.
[12] Aymar, R., Böhmer, H., Koechlin, F., Proceedings of the VIth European Conference on Controlled Fusion and Plasma Physics, vol. II, 422 (Moscou, 1973).

[13] Daughney, C. C., Holmes, L. S., Paul, J. W., Phys. Rev. Lett. 25 (1970) 497.

[14] Mazzucatto, E., Phys. Rev. Lett. 36 (1976) 792.

[15] EQuIPE T. F. R., Proceedings of the VIth International Conference on Plasma Physics and Controlled Nuclear Fusion Research, Berchtesgaden, 1 (1976) 35 (International Atomic Energy Agency, Vienna, 1977).

[16] Voir par exemple : Bekefi, G., Radiation Processes in Plasmas (J. Wiley, New York) 1966.

[17] Voir aussi Shefrield, J., Plasma Scattering of Electromagnetic Radiation (Academic Press, London) 1975.

[18] T. F. R., Group, Proceedings of the VIth European Conference on Controlled Fusion and Plasma Physics, 2 (1973) 20 (Joint Institute for Nuclear Research, Moscou, 1973).

[19] Goldston, R. J., Mazzucato, E., Slusher, R. E., Surko, C. M., même référence que [15], 1, 371.

[20] WorT, D. J., Culham Laboratory Report CLM-R27 (1963).

[21] Cox, J. R., Plasma Phys. 18 (1976) 67. 